\title{
MODEL-THEORETIC ASPECTS OF THE GURARIJ OPERATOR SYSTEM
}

\author{
ISAAC GOLDBRING AND MARTINO LUPINI
}

\begin{abstract}
We establish some of the basic model theoretic facts about the Gurarij operator system $\mathbb{G S}$ recently constructed by the second-named author. In particular, we show: (1) $\mathbb{G S}$ is the unique separable 1-exact existentially closed operator system; (2) $\mathbb{G S}$ is the unique separable nuclear model of its theory; (3) every embedding of $\mathbb{G S}$ into its ultrapower is elementary; (4) $\mathbb{G S}$ is the prime model of its theory; and (5) $\mathbb{G S}$ does not have quantifier-elimination, whence the theory of operator systems does not have a model companion. We also show that, for any $q \in \mathbb{N}$, the theories of $M_{q}$-spaces and $M_{q}$-systems do have a model companion, namely the Fraïssé limit of the class of finite-dimensional $M_{q}$-spaces and $M_{q}$-systems respectively; moreover we show that the model companion is separably categorical. We conclude the paper by showing that no $\mathrm{C}^{*}$ algebra can be existentially closed as an operator system.
\end{abstract}

\section{INTRODUCTION}

The Gurarij Banach space $\mathbb{G}$ is a Banach space first constructed by Gurarij in [15]. It has the following universal property: whenever $X \subseteq Y$ are finite-dimensional Banach spaces, $\phi: X \rightarrow \mathbb{G}$ is a linear isometry, and $\epsilon>0$, there is an injective linear map $\psi: Y \rightarrow \mathbb{G}$ extending $\phi$ such that $\|\psi\|\left\|\psi^{-1}\right\|<1+\epsilon$. The uniqueness of such a space was first proved by Lusky in [26] and later a short proof was given by Kubis and Solecki in [21].

Model-theoretically, $\mathbb{G}$ is a relatively nice object. Indeed, Ben Yaacov [1] showed that $\mathbb{G}$ is the Fraïssé limit of the class of finite-dimensional Banach spaces (yielding yet another proof of the uniqueness of $\mathbb{G}$ ). Moreover, Ben Yaacov and Henson [3] showed that the theory of $\mathbb{G}$ is separably categorical and admits quantifier-elimination; since every separable Banach space embeds in $\mathbb{G}$, it follows that the theory of $\mathbb{G}$ is the modelcompletion of the theory of Banach spaces. (On the other hand, it is folklore that the theory of $\mathbb{G}$ is unstable, so perhaps the nice model-theoretic properties of $\mathbb{G}$ end here.)

In [27], Oikhberg introduced a noncommutative analog of $\mathbb{G}$ which he referred to as (no surprise) a noncommutative Gurarij operator space. Here, "noncommutative" refers to the fact that we are considering operator spaces, the noncommutative analog of Banach spaces. (In Section 2, a primer on operator spaces - amongst other things - will be given.) A Gurarij operator space satisfies the noncommutative analog of the defining property of $\mathbb{G}$ mentioned above, where the completely bounded norm replaces the usual norm of linear maps and the finite-dimensional spaces are further assumed to be 1-exact. Approximate uniqueness of a Gurarij operator space was proven by Oikhberg in [27].

Precise uniqueness of the Gurarij operator space was later proven in [23] by realizing the Gurarij operator space (henceforth referred to as $\mathbb{N} \mathbb{G}$ ) as the Fraïssé limit of the class of finite-dimensional 1-exact operator spaces. In [24], the second author established the existence and uniqueness of the Guarij operator system $\mathbb{G S}$, which is the Fraïsé limit

Goldbring's work was partially supported by NSF CAREER grant DMS-1349399. Lupini's work was supported by the York University Susan Mann Dissertation Scholarship and by the ERC Starting grant no. 259527 of Goulnara Arzhantseva. This work was initiated during a visit of the second author to the University of Illinois at Chicago. The hospitality of the UIC Mathematics Department is gratefully acknowledged. 
of the class of finite-dimensional 1-exact operator systems. In this paper, we establish some of the main model-theoretic facts about $\mathbb{G S}$. We summarize these as follows:

\section{Theorem 1.1.}

(1) $\mathbb{G S}$ is the unique separable 1-exact existentially closed operator system;

(2) $\mathbb{G S}$ is the unique separable nuclear model of its theory;

(3) every embedding of $\mathbb{G S}$ into its ultrapower is elementary;

(4) $\mathbb{G S}$ is the prime model of its theory;

(5) $\mathbb{G S}$ does not have quantifier-elimination, whence the theory of operator systems does not have a model companion.

We do not know whether the analogous statements hold for $\mathbb{N} G$. The proofs in the operator system case rely strongly on Choi's characterization of completely positive linear maps with domain $\mathbb{M}_{n}$ [10]; see also [29, Theorem 3.14]. Such a characterization has no counterpart for (not necessarily unital) completely bounded maps, as pointed out in [29, page 114].

The notion of $M_{q}$-space is an intermediate generalization of Banach spaces where only matrix norms up to order $q$ are considered; likewise, there is the notion of $M_{q}$-system (also called $q$-minimal operator system). The classes of finite-dimensional $M_{q}$-spaces (resp. $M_{q}$-systems) have Frïssé limits, called $\mathbb{G}_{q}$ (resp. $\mathbb{G}_{q}^{u}$ ). We show that the theory of $\mathbb{G}_{q}$ (resp. the theory of $\mathbb{G}_{q}^{u}$ ) is the model-completion of the theory of $M_{q}$-spaces (resp. $M_{q}$-systems) and is separably categorical.

We conclude the paper by proving that no existentially closed operator system can be completely order isomorphic to a $\mathrm{C}^{*}$-algebra. While this fact was proven for $\mathbb{G S}$ itself in [24], our proof here is somewhat more elementary and covers all existentially closed operator systems.

We assume that the reader is familiar with continuous logic as it pertains to operator algebras (see 12] for a good primer). In the following all the quantifiers are supposed to range in the unit ball. If $X$ is an operator space or an operator system, its closed unit ball is denoted by Ball $(X)$. In Section 2, we describe all of the necessary background on operator spaces and operator systems.

Acknowledgments. We would like to thank Timur Oikhberg for pointing out a mistake in the original version of this paper.

\section{Preliminaries}

2.1. Operator spaces and $M_{q}$-spaces. If $H$ is a Hilbert space, let $B(H)$ denote the space of bounded linear operators on $H$ endowed with the pointwise linear operations and the operator norm. One can identify $M_{n}(B(H))$ with the space $B\left(H^{\oplus n}\right)$, where $H^{\oplus n}$ is the $n$-fold Hilbertian sum of $H$ with itself. A (concrete) operator space is a closed subspace of $B(H)$. If $X$ is an operator space, then the inclusion $M_{n}(X) \subset M_{n}(B(H))$ induces a norm on $M_{n}(X)$ for every $n \in \mathbb{N}$. If $X, Y$ are operator spaces, $\phi: X \rightarrow Y$ is a linear map, and $n \in \mathbb{N}$, then the $n$-th amplification $\phi^{(n)}: M_{n}(X) \rightarrow M_{n}(Y)$ is defined by

$$
\left[x_{i j}\right] \mapsto\left[\phi\left(x_{i j}\right)\right]
$$

A linear map $\phi$ is completely bounded if $\sup _{n}\left\|\phi^{(n)}\right\|<+\infty$, in which case one defines the completely bounded norm $\|\phi\|_{c b}:=\sup _{n}\left\|\phi^{(n)}\right\|$. We say that $\phi$ is completely contractive if $\phi^{(n)}$ is contractive for every $n \in \mathbb{N}$ and completely isometric if $\phi^{(n)}$ is isometric for every $n \in \mathbb{N}$.

If $q \in \mathbb{N}, \alpha, \beta \in M_{q}$, and $x \in M_{q}(X)$ we denote by $\alpha . x . \beta$ the element of $M_{q}(X)$ obtained by taking the usual matrix product. The matrix norms on an operator space 
satisfy the following relations, known as Ruan's axioms: for every $q, k \in \mathbb{N}$ and $x \in$ $M_{q}(X)$ we have

$$
\left\|\left[\begin{array}{ll}
x & 0 \\
0 & 0
\end{array}\right]\right\|_{M_{q+k}(X)}=\|x\|_{M_{q}(X)},
$$

and for every $q, n \in \mathbb{N}, \alpha_{i}, \beta_{i} \in M_{q}$ and $x_{i} \in M_{q}(X)$ for $i=1,2, \ldots, n$, we have

$$
\left\|\sum_{i=1}^{n} \alpha_{i} \cdot x_{i} \cdot \beta_{i}\right\| \leq\left\|\sum_{i=1}^{n} \alpha_{i} \alpha_{i}^{*}\right\| \max _{1 \leq i \leq n}\left\|x_{i}\right\|\left\|\sum_{i=1}^{n} \beta_{i}^{*} \beta_{i}\right\|,
$$

where $\alpha_{i} \cdot x_{i} \cdot \beta_{i}$ denotes the usual matrix multiplication. Ruan's theorem [31] asserts that, conversely, any matricially normed vector space $X$ with matrix norms satisfying Ruan's axioms is linearly completely isometric to a subspace of $B(H)$; see also [30, $\S 2.2]$. We will regard operator spaces as structures in the language for operator spaces $\mathcal{L}_{\text {ops }}$ introduced in [13, Appendix B]. It is clear that the class of operator spaces, viewed as $\mathcal{L}_{\text {ops }}$-structures, forms an axiomatizable class by semantic considerations [12, §2.3.2]. Using Ruan's theorem, concrete axioms for the class of operator spaces are given in [13, Theorem B.3].

A finite-dimensional operator space $X$ is said to be 1-exact if there are natural numbers $k_{n}$ and injective linear maps $\phi_{n}: X \rightarrow M_{k_{n}}$ such that $\left\|\phi_{n}\right\|_{c b}\left\|\phi_{n}^{-1}\right\|_{c b} \rightarrow 1$ as $n \rightarrow \infty$. An arbitrary operator space is 1-exact if all its finite-dimensional subspaces are 1-exact. It is well known that a $\mathrm{C}^{*}$-algebra is exact if and only if it is 1-exact when viewed as an operator space. We mention in passing that the 1-exact operator spaces do not form an axiomatizable class, even amongst the separable ones.

For $q \in \mathbb{N}$, an $M_{q}$-space is a vector space $X$ such that $M_{q}(X)$ is endowed with a norm satisfying Equation (2.1) for every $n \in \mathbb{N}, \alpha_{i}, \beta_{i} \in M_{q}$, and $x_{i} \in M_{q}(X)$ for $i=1,2, \ldots, n$. Clearly an $M_{q}$-space is canonically an $M_{n}$-space for $n \leq q$ via the upperleft corner embedding of $M_{n}(X)$ into $M_{q}(X)$. Let $T_{M_{q}}$ be the reduct of the language of operator spaces where only the sorts for $M_{n, m}$ for $n, m \leq 2 q$ and $M_{n, m}(X)$ for $n, m \leq q$ are retained. Once again, by syntactic considerations, it is straightforward to verify that $M_{q}$-spaces form an axiomatizable class in the language $T_{M_{q}}$. One can write down explicit axioms using Equation (2.1).

If $\phi: X \rightarrow Y$ is a linear map between $M_{q}$-spaces, then $\phi$ is said to be $q$-bounded if $\phi^{(q)}: M_{q}(X) \rightarrow M_{q}(X)$ is bounded. In such a case one sets $\|\phi\|_{q}=\left\|\phi^{(q)}\right\|$. A linear map $\phi$ is then said to be $q$-contractive if $\phi^{(q)}$ is contractive and $q$-isometric if $\phi^{(q)}$ is isometric.

It is shown in [22, Théorème I.1.9] that any $M_{q}$-space can be concretely represented as a subspace of $C\left(K, M_{q}\right)$ for some compact Hausdorff space $K$. Here $C\left(K, M_{q}\right)$ is the space of continuous functions from $K$ to $M_{q}$ endowed with the $M_{q}$-space structure obtained by canonically identifying $M_{q}\left(C\left(K, M_{q}\right)\right)$ with $C\left(K, M_{q} \otimes M_{q}\right)$, where the latter is endowed with the uniform norm.

An $M_{q}$-space $X$ admits a canonical operator space structure denoted by $\operatorname{MIN}_{q}(X)$ [22, I.3]. The corresponding operator norms are defined by

$$
\|x\|=\sup _{\phi}\left\|\phi^{(q)}(x)\right\|
$$

for $n \in \mathbb{N}$ and $x \in M_{n}(X)$, where $\phi$ ranges over all $q$-contractive linear maps $\phi$ : $X \rightarrow M_{q}$. The $\operatorname{MIN}_{q}$ operator space structure on $X$ is characterized by the following property: the identity map $X \rightarrow \operatorname{MIN}_{q}(X)$ is a $q$-isometry, and for any operator space $Y$ and linear map $\phi: Y \rightarrow X$, the map $\phi$ is $q$-bounded if and only if $\phi: Y \rightarrow \operatorname{MIN}_{q}(X)$ is completely bounded, in which case $\|\phi: Y \rightarrow X\|_{q}=\left\|\phi: Y \rightarrow \operatorname{MIN}_{q}(X)\right\|$. 
We will call an operator space of the form $\operatorname{MIN}_{q}(X)$ a $\operatorname{MIN}_{q^{-}}$space. It is clear that semantically there is really no difference between $M_{q}$-spaces and $\mathrm{MIN}_{q}$-spaces. However there is a syntactical difference between these two notions as they correspond to regarding these spaces as structures in two different languages. We will therefore retain the two distinct names to avoid confusion.

It follows from the characterizing property of the functor $\mathrm{MIN}_{q}$ that $\mathrm{MIN}_{q}$-spaces are closed under subspaces, isomorphism, and ultraproducts. (For the latter, one needs to observe that the ultraproduct of a family of $q$-bounded maps from $X$ to $M_{q}$ is again a $q$-bounded map from $X$ to $M_{q}$.) Therefore, $\mathrm{MIN}_{q}$-spaces form an axiomatizable class in the language of operator spaces. Furthermore the functor $\mathrm{MIN}_{q}$ is an equivalence of categories from $M_{q}$-spaces to $\mathrm{MIN}_{q}$-spaces. It follows from Beth's definability theorem $[12, \S 3.4]$ the that the matrix norms on $M_{n}(X)$ for $n>q$ are definable in the language of $M_{q^{-}}$-spaces.

2.2. Operator systems and $M_{q}$-systems. Suppose that $X$ is an operator space. An element $u \in X$ is a unitary if there is a linear complete isometry $\phi: X \rightarrow B(H)$ such that $\phi(u)$ is the identity operator on $H$. It is shown in [8] that if $X$ is a $\mathrm{C}^{*}$-algebra, then this corresponds with the usual notion of unitary. Theorem 2.4 of [8] provides the following abstract characterization of unitaries: $u$ is a unitary of $X$ if an only if, for every $n \in \mathbb{N}$ and $x \in M_{n}(X)$, one has that

$$
\left\|\left[\begin{array}{ll}
u_{n} & x
\end{array}\right]\right\|^{2}=\left\|\left[\begin{array}{c}
u_{n} \\
x
\end{array}\right]\right\|^{2}=1+\|x\|^{2},
$$

where $u_{n}$ denotes the diagonal matrix in $M_{n}(X)$ with $u$ in the diagonal entries. A unital operator space is an operator space with a distinguished unitary. The abstract characterization of unitaries shows that unital operator spaces form an axiomatizable class in the language $T_{\text {uosp }}$ obtained by adding to the language of operator spaces a constant symbol for the unit.

If $X$ is an $M_{q}$-space, then we say that an element $u$ of $M_{q}$ is a unitary if there is a linear $q$-isometry $\phi: X \rightarrow C\left(K, M_{q}\right)$ mapping the distinguished unitary to the function constantly equal to the identity of $M_{q}$. Observe that $u$ is a unitary of $X$ if and only if it is a unitary of $\operatorname{MIN}_{q}(X)$. In fact, if $u$ is a unitary of $X$ and $\phi: X \rightarrow C\left(K, M_{q}\right)$ is a unital linear $q$-isometry, then $\phi: \operatorname{MIN}_{q}(X) \rightarrow C\left(K, M_{q}\right)$ is a unital complete isometry. If $\psi: C\left(K, M_{q}\right) \rightarrow B(H)$ is a unital complete isometry, then $\phi \circ \psi$ witnesses the fact that $u$ is a unitary of $\operatorname{MIN}_{q}(X)$. Conversely suppose that $u$ is a unitary of $\operatorname{MIN}_{q}(X)$. It follows from the universal property that characterizes the injective envelope of an operator space [7, $\S 4.3]$ that the injective envelope $I\left(\operatorname{MIN}_{q}(X)\right)$ is a $\operatorname{MIN}_{q}$-space. Since the $\mathrm{C}^{*}$-envelope $C_{e}^{*}\left(\operatorname{MIN}_{q}(X), u\right)$ of the unital operator space $\operatorname{MIN}_{q}(X)$ with unit $u$ can be realized as a subspace of $I\left(\operatorname{MIN}_{q}(X)\right)$ by [7, §4.3], it follows that $C_{e}^{*}\left(\operatorname{MIN}_{q}(X), u\right)$ is an $M_{q}$-space. Equivalently $C_{e}^{*}\left(\operatorname{MIN}_{q}(X), u\right)$ is a $q$-subhomogeneous $\mathrm{C}^{*}$-algebra [6, IV.1.4.1]. Therefore there is an injective unital *homomorphism $\psi: C_{e}^{*}\left(\operatorname{MIN}_{q}(X), u\right) \rightarrow \bigoplus_{k \leq q} C\left(K, M_{k}\right)$ for some compact Hausdorff space $K$; see [6, IV.1.4.3].

Moreover the proof of [8, Theorem 2.4] shows that an element $u$ of an $M_{q}$-space $X$ is a unitary if and only if

$$
\left\|\left[\begin{array}{ll}
u_{q} & x
\end{array}\right]\right\|_{M_{2 q}\left(\operatorname{MIN}_{q}(X)\right)}^{2}=\left\|\left[\begin{array}{c}
u_{q} \\
x
\end{array}\right]\right\|_{M_{2 q}\left(\operatorname{MIN}_{q}(X)\right)}^{2}=1+\|x\|^{2} .
$$

A unital $M_{q}$-space is an $M_{q}$-space with a distinguished unitary. Let $T_{u M_{q}}$ the language of $M_{q}$-spaces with an additional constant symbol for the distinguished unitary. Then the abstract characterization of unitaries in $M_{q}$-spaces provided above together with 
the fact that the matrix norms on $\operatorname{MIN}_{q}(X)$ are definable show that unital $M_{q}$-spaces form an axiomatizable class in the language of unital $M_{q}$-spaces.

An operator system is a unital operator space $(X, 1)$ such that there exists a linear complete isometry $\phi: X \rightarrow B(H)$ with $\phi(1)=1$ and $\phi[X]$ a self-adjoint subspace of $B(H)$. By [8, Theorem 3.4], a unital operator space is an operator system if and only if for every $n \in \mathbb{N}$ and for every $x \in X$ there is $y \in Y$ such that $\|y\| \leq\|x\|$ and

$$
\left\|\left[\begin{array}{cc}
n 1 & x \\
y & n 1
\end{array}\right]\right\|^{2} \leq 1+n^{2} .
$$

This shows that operator systems form an axiomatizable class in the language of unital operator spaces.

The representation of an operator system $X$ as a unital self-adjoint subspace of $X$ induces on $X$ an involution $x \mapsto x^{*}$ and positive cones on $M_{n}(X)$ for every $n \in \mathbb{N}$. A linear map between operator systems is positive if it maps positive elements to positive elements, and completely positive if all its amplifications are positive. In the following we will abbreviate "unital completely positive" with ucp. A unital linear map between operator systems is completely positive if and only if it is completely contractive, and in such a case it is necessarily self-adjoint. Therefore by Beth's definability theorem again, the involution and the positive cones are definable. Explicitly $x \in M_{n}(X)$ is positive if and only if

$$
\left[\begin{array}{cc}
1_{n} & x \\
x & 1_{n}
\end{array}\right]
$$

has norm at most 1 [29, Lemma 3.1]. Moreover the adjoint of $x$ is the element $y$ of $X$ that minimizes the left-hand side of Equation 2.2. An alternative axiomatization of operator systems in terms of the unit, the involution, and the positive cones is suggested in [13, Appendix B]. Since in turn the matrix norms are definable from these items, these two axiomatizations are equivalent.

The operator system analog $\mathrm{OMIN}_{q}$ of $\mathrm{MIN}_{q}$ has been introduced and studied in [33]. It is shown there that $\mathrm{OMIN}_{q}$ has entirely analogous properties as $\mathrm{MIN}_{q}$, when one replaces operator spaces with operator systems, and (complete) contractions with unital (completely) positive maps. An $M_{q}$-system (also called $q$-minimal operator system in [33] ) is a unital $M_{q}$-space $X$ such there is a unital $q$-isometry $\phi: X \rightarrow C\left(K, M_{q}\right)$ such that the image of $\phi$ is a self-adjoint subspace of $C\left(K, M_{q}\right)$. Equivalently, $X$ is an $M_{q^{-}}$ system if and only if $X$ is a unital $M_{q}$-space such that $\operatorname{MIN}_{q}(X)$ is an operator system. The above axiomatizations of operator systems in the language of unital operator spaces and of unital $M_{q}$-spaces in the language of unital $M_{q}$-spaces show that $M_{q}$-systems are axiomatizable in the language of unital $M_{q}$-spaces. Again Beth's definability theorem shows that the all the matrix norms as well as the positive cones and the involution are definable.

2.3. $\mathbb{G}_{q}, \mathbb{G}_{q}^{u}, \mathbb{N} \mathbb{G}$, and $\mathbb{G S}$. It is shown in $[23, \S 3]$ that the class of finite-dimensional $M_{q}$-spaces is a Fraïssé class in the sense of [1]. The corresponding Fraïssé limit $\mathbb{G}_{q}$ is a separable $M_{q}$-space that is characterized by the following property: whenever $E \subset F$ are finite-dimensional $M_{q}$-spaces, $f: E \rightarrow \mathbb{G}_{q}$ is a linear $q$-isometry, and $\varepsilon>0$, then there is a linear extension $g: F \rightarrow \mathbb{G}_{q}$ of $f$ such that $\|g\|_{q}\left\|g^{-1}\right\|_{q} \leq 1+\varepsilon$; see [23, §3.3]. Similarly, it is shown in [24, $§ 4.5]$ that finite-dimensional $M_{q}$-systems form a Fraïssé class. The corresponding limit is a separable $M_{q}$-system $\mathbb{G}_{q}^{u}$ which is characterized by the same property as $\mathbb{G}_{q}$, where one replaces operator spaces with operator systems and linear maps with unital linear maps; see [24, Proposition 4.9].

The class of finite-dimensional 1-exact operator spaces is shown to be a Fraïssé class in [23, §4]. The corresponding limit is the (noncommutative) Gurarij operator space $\mathbb{N G}$, characterized by the property that given finite-dimensional 1-exact operator spaces 
$E \subset F$, a linear complete isometry $\phi: E \rightarrow \mathbb{N} \mathbb{G}$, and $\varepsilon>0$, there exists an injective linear map $\widehat{\phi}: F \rightarrow \mathbb{N} G$ extending $\phi$ such that $\|\widehat{\phi}\|_{c b}\left\|\widehat{\phi}^{-1}\right\|_{c b} \leq 1+\varepsilon$. Similarly the class of finite-dimensional 1-exact operator systems is shown to be a Fraïssé class in [24, §3]. The corresponding limit is the Gurarij operator space $\mathbb{G S}$, characterized by a similar property as $\mathbb{N} \mathbb{G}$ where one replaces operator spaces with operator systems and linear maps with unital linear maps.

At this point, it is worth recording the following:

Proposition 2.1. Both $\mathrm{Th}(\mathbb{N} \mathbb{G})$ and $\mathrm{Th}(\mathbb{G S})$ have a continuum of nonisomorphic separable models.

Proof. Every operator space admits a completely isometric embedding into $\prod_{\mathcal{U}} M_{n}$, the operator space ultraproduct of matrix algebras. Furthermore $M_{n}$ admits a completely isometric embedding into $\mathbb{N} \mathbb{G}$ by universality. Henceforth any separable operator space embeds into a separable model of the theory of $\mathbb{N G}$. Suppose now, towards a contradiction, that $\kappa<\mathfrak{c}$ and $\left(Z_{i}\right)_{i<\kappa}$ enumerate all of the separable models of the theory of $\mathbb{N} G$ up to complete isometry. Let $Z=\bigoplus_{i<\kappa} Z_{i}$. If $X$ is any separable operator space, then $X$ embeds into some $Z_{i}$ and hence embeds into $Z$. It follows that $Z$ is an operator space of density character $\kappa$ that contains all separable operator spaces. This contradicts the fact that for $n \geq 3$ the space of $n$-dimensional operator spaces has density character $\mathfrak{c}$ with respect to the completely bounded distance, which is the main result of [19] as formulated in [30], Corollary 21.15 and subsequent remark. The assertion about $\mathbb{G S}$ can be proved in the same way, after observing that any separable operator space embeds into a separable operator system.

Let $\operatorname{Aut}(\mathbb{N G})$ be the automorphism group of $\mathbb{N} \mathbb{G}$, i.e. the group of surjective linear complete isometries $\alpha: \mathbb{N G} \rightarrow \mathbb{N G}$, endowed with the topology of pointwise convergence. The homogeneity property of $\mathbb{N} G$ implies that every point in the unit sphere of $\mathbb{N} \mathbb{G}$ has dense orbit under the action of Aut $(\mathbb{N} \mathbb{G})$. Therefore it follows from Proposition 2.1 and [4, Theorem 2.4] that Aut $(\mathbb{N} \mathbb{G})$ is not Roelcke precompact; see [4, Definition 1.1].

\section{The operator spaces $\mathbb{G}_{q}$ And the operator systems $\mathbb{G}_{q}^{u}$}

3.1. The operator spaces $\mathbb{G}_{q}$. The following amalgamation result is proved in 23 , Lemma 3.1]; see also [25, Lemma 2.1].

Lemma 3.1. If $X \subset \widehat{X}$ and $Y$ are $M_{q}$-spaces, and $f: X \rightarrow Y$ is a linear injective $q$-contraction such that $\left\|f^{-1}\right\|_{q} \leq 1+\delta$, then there exists an $M_{q}$-space $Z$ and $q$-isometric linear maps $i: \widehat{X} \rightarrow Z$ and $j: Y \rightarrow Z$ such that $\left\|i_{\mid X}-j \circ f\right\|_{q} \leq \delta$.

Arguing as in the proof of [21, Theorem 1.1], where [21, Lemma 2.1] is replaced by Lemma 3.1. shows that $\mathbb{G}_{q}$ has following homogeneity property: whenever $X$ is a finitedimensional subspace of $\mathbb{G}_{q}$ and $\phi: X \rightarrow \mathbb{G S}$ is a linear map such that $\|\phi\|_{q}<1+\delta$ and $\left\|\phi^{-1}\right\|_{q}<1+\delta$, there exists a linear surjective $q$-isometry $\alpha: \mathbb{G}_{q} \rightarrow \mathbb{G}_{q}$ such that $\left\|\alpha_{\mid X}-\phi\right\|_{q}<\delta$.

\section{Proposition 3.2. $\operatorname{Th}\left(\mathbb{G}_{q}\right)$ is separably categorical.}

Proof. Suppose that $E \subset F$ are finite-dimensional $M_{q}$-spaces, where $E$ has dimension $k$ and $F$ has dimension $m>k$. Fix also a normalized basis $\vec{a}=\left(a_{1}, \ldots, a_{m}\right)$ of $F$ such that $\left(a_{1}, \ldots, a_{k}\right)$ is a basis of $E$. For $1 \leq n \leq m$ we let $X_{n}$ denote those $n$-tuples $\left(\alpha_{1}, \ldots, \alpha_{n}\right)$ from $M_{q}$ such that

$$
\left\|\sum_{i=1}^{n} \alpha_{i} \otimes e_{i}\right\|=1 .
$$


Note that $X_{n}$ is a compact subset of $M_{q}^{n}$, whence definable. We then let $\eta_{\vec{a}, n}\left(x_{1}, \ldots, x_{n}\right)$ denote the formula

$$
\sup _{\left(\alpha_{1}, \ldots, \alpha_{n}\right) \in X_{n}} \max \left\{\left\|\sum_{i=1}^{n} \alpha_{i} \otimes x_{i}\right\| \dot{-1,1} \cdot\left\|\sum_{i=1}^{n} \alpha_{i} \otimes x_{i}\right\|\right\}
$$

where $a-b$ denotes the maximum of $a-b$ and 0 . For the sake of brevity, we write $\eta_{\vec{a}, n}(\vec{x})$ instead of $\eta_{\vec{a}, n}\left(x_{1}, \ldots, x_{n}\right)$; no confusion should arise as the subscript indicates what the free variables are. Furthermore define $\theta_{\vec{a}, k}\left(x_{1}, \ldots, x_{k}, y_{1}, \ldots, y_{m}\right)$ to be the formula

$$
\max \left\{\eta_{\vec{a}, m}(\vec{y}), \sup _{\left(\beta_{1}, \ldots, \beta_{k}\right) \in X_{k}}\left\|\sum_{i=1}^{k} \beta_{i} \otimes\left(x_{i}-y_{i}\right)\right\|-2 \eta_{\vec{a}, k}(\vec{x})\right\} .
$$

We now let $\sigma_{\vec{a}, k}$ denote the sentence

$$
\sup _{x_{1}, \ldots, x_{k}} \min \left\{\frac{1}{4}-\eta_{\vec{a}, k}(\vec{x}), \inf _{y_{1}, \ldots, y_{m}} \theta_{\vec{a}, k}(\vec{x}, \vec{y})\right\} .
$$

Claim 1: $\sigma_{\vec{a}, k}^{\mathbb{G}_{q}}=0$.

Proof of Claim 1: Suppose that $b_{1}, \ldots, b_{k}$ are elements in the unit ball of $\mathbb{G}_{q}$ such that $\eta_{\vec{a}, k}(\vec{b})<\frac{1}{4}$. Fix $\delta \in\left(0, \frac{1}{4}\right]$ such that $\eta_{\vec{a}, k}(\vec{b})<\delta$. Define the linear map $f: E \rightarrow \mathbb{G}_{q}$ by $f\left(a_{i}\right)=b_{i}$ for $i \leq k$. Observe that $\|f\|_{q}<1+\delta$ and $\left\|f^{-1}\right\|_{q}<1+2 \delta$. Therefore by the above mentioned homogeneity property of $\mathbb{G}_{q}$ there exists a linear $q$-isometry $q: F \rightarrow \mathbb{G}_{q}$ such that $\left\|g_{\mid E}-f\right\|_{q}<2 \delta$. Let $c_{i}=g\left(a_{i}\right)$ for $1 \leq i \leq m$ and observe that $\theta(\vec{b}, \vec{c})=0$.

Claim 2: If $Z$ is a separable $M_{q}$-space for which $\sigma_{\vec{a}, k}^{Z}=0$ for each $k<m$ and $\vec{a}$ as above, then $Z$ is $q$-isometric to $\mathbb{G}_{q}$.

Proof of Claim 2: Suppose that $f: E \rightarrow Z$ is a linear $q$-isometry, $\operatorname{dim}(E)=k, F$ is an $m$-dimensional $M_{q}$-space containing $E$, and $\varepsilon>0$ is given. Fix a normalized basis $\vec{a}=\left(a_{1}, \ldots, a_{m}\right)$ of $F$ for which $a_{1}, \ldots, a_{k}$ is a basis of $E$, and $\eta \in\left(0, \frac{1}{4}\right)$ small enough. Set $b_{i}=f\left(a_{i}\right)$ for $i \leq k$. Since $\eta_{\vec{a}, k}(\vec{b})=0$ and $\sigma_{\vec{a}, k}^{Z}=0$, there are $c_{i} \in Z$ for $1 \leq i \leq m$ such that $\theta^{Z}(\vec{b}, \vec{c}) \leq \eta$. Therefore the linear map $g: F \rightarrow \mathbb{G}_{q}$ defined by $g\left(a_{i}\right)=c_{i}$ for $1 \leq i \leq m$ is such that $\|g\|_{q} \leq 1+\eta,\left\|g^{-1}\right\|_{q} \leq 1+2 \eta$, and $\left\|g_{\mid E}-f\right\|_{q} \leq 1+\eta$. The "small perturbation argument" - see [9, Lemma 12.3.15] and also [30, §2.13] —allows one to perturb $g$ to a linear map that extends $f$ while only slighlty changing the $q$-norms of $g$ and its inverse. Upon choosing $\eta$ small enough, this shows that $Z$ satisfies the approximate homogeneity property that characterizes $\mathbb{G}_{q}$.

We now give an alternate proof of the preceding theorem using the Ryll-Nardzewski Theorem [2, Theorem 12.10]. For the definition of approximately oligomorphic action, see [4, Definition 2.1].

Proposition 3.3. Suppose that $q \in \mathbb{N}$. Then the action of Aut $\left(\mathbb{G}_{q}\right)$ on the unit ball Ball $\left(\mathbb{G}_{q}\right)$ of $\mathbb{G}_{q}$ is approximately oligomorphic.

Proof. Observe that the quotient space Ball $\left(\mathbb{G}_{q}\right) / / \operatorname{Aut}\left(\mathbb{G}_{q}\right)$ is isometric to $[0,1]$ and hence compact. We need to show that the quotient space Ball $\left(\mathbb{G}_{q}\right)^{k} / / \operatorname{Aut}\left(\mathbb{G}_{q}\right)$ is compact for every $k \in \mathbb{N}$. This is essentially shown in [23, Proposition 3.5]. We denote by $\left[a_{1}, \ldots, a_{k}\right]$ the image of the tuple $\left(a_{1}, \ldots, a_{k}\right)$ of Ball $\left(\mathbb{G}_{q}\right)^{k}$ in the quotient $\operatorname{Ball}\left(\mathbb{G}_{q}\right)^{k} / / \operatorname{Aut}\left(\mathbb{G}_{q}\right)$. Suppose that $\left[a_{1}^{(n)}, \ldots, a_{k}^{(n)}\right]$ is a sequence in Ball $\left(\mathbb{G}_{q}\right)^{k} / / \operatorname{Aut}\left(\mathbb{G}_{q}\right)$. After passing to a subsequence we can assume that, for every $\alpha_{1}, \ldots, \alpha_{k} \in M_{q}$ the sequence

$$
\left\|\alpha_{1} \otimes a_{1}^{(n)}+\cdots+\alpha_{n} \otimes a_{k}^{(n)}\right\|
$$


converges. This implies that the convergence is uniform on the unit ball of $M_{q}$. Suppose that $a_{1}, \ldots, a_{k} \in \mathbb{G}_{q}$ are such that

$$
\left\|\alpha_{1} \otimes a_{1}+\cdots+\alpha_{n} \otimes a_{k}\right\|=\lim _{n}\left\|\alpha_{1} \otimes a_{1}^{(n)}+\cdots+\alpha_{n} \otimes a_{k}^{(n)}\right\| .
$$

Then [23, Proposition 3.4] shows that $\left[a_{1}, \ldots, a_{k}\right]$ is the limit of

$$
\left(\left[a_{1}^{(n)}, \ldots, a_{k}^{(n)}\right]\right)_{n \in \mathbb{N}}
$$

in Ball $\left(\mathbb{G}_{q}\right)^{k} / /$ Aut $\left(\mathbb{G}_{q}\right)$. This shows that every sequence has a convergent subsequence and hence such a space is compact.

It follows from Proposition 3.2 and [4, Theorem 2.4] that the automorphism group Aut $\left(\mathbb{G}_{q}\right)$, i.e. the group of surjective $q$-isometric linear maps $\alpha: \mathbb{G}_{q} \rightarrow \mathbb{G}_{q}$ is Roelcke precompact for every $q \in \mathbb{N}$. The Roelcke compactification of a Roelcke precompact group is described model-theoretically in [4, §2.2].

3.2. Quantifier-elimination. Recall from [2, Proposition 13.2] the following test for quantifier-elimination:

Fact 3.4. Suppose that, whenever $\mathcal{M}, \mathcal{N} \models T, \mathcal{M}_{0}, \mathcal{N}_{0}$ are finitely generated substructures of $\mathcal{M}$ and $\mathcal{N}$ respectively, $\Phi: \mathcal{M}_{0} \rightarrow \mathcal{N}_{0}$ is an isomorphism, $\varphi(\vec{x})$ is an $\mathcal{L}$-formula, and $\vec{a} \in \mathcal{M}_{0}$, we have

$$
\varphi^{\mathcal{M}}(\vec{a})=\varphi^{\mathcal{N}}(\Phi(\vec{a}))
$$

Then $T$ admits quantifier-elimination.

Proposition 3.5. $\operatorname{Th}\left(\mathbb{G}_{q}\right)$ has quantifier-elimination.

Proof. This follows immediately from the above quantifier-elimination test and the homogeneity and separable categoricity of $\mathbb{G}_{q}$.

3.3. The operator systems $\mathbb{G}_{q}^{u}$. Proceeding as in Subsection 3.1, and using the characterization of $\mathbb{G}_{q}^{u}$ given in $[24, \S 4.5]$, one can prove similarly as above the following facts.

Proposition 3.6. $\operatorname{Th}\left(\mathbb{G}_{q}^{u}\right)$ is separably categorical

Corollary 3.7. $\operatorname{Th}\left(\mathbb{G}_{q}^{u}\right)$ has quantifier-elimination.

Remark. One can also prove the analogue for $\mathbb{G}_{a}^{u}$ of Proposition 3.3. In this case one needs to use results from [24] and in particular [24, Lemma 3.8].

\section{The Guarij operator System $\mathbb{G S}$}

In this section, we establish the model theoretic properties of the Gurarij operator system announced in the introduction. We first need an introductory subsection on lifting ucp maps.

\subsection{Perturbation and ucp maps.}

Lemma 4.1. Suppose that $X$ and $Y$ are operator systems and $\phi: X \rightarrow Y$ is a unital linear map such that $\|\phi\| \leq 1+\delta$. If $x \in \operatorname{Ball}(X)$ is self-adjoint, then $\|\operatorname{Im}(\phi(x))\| \leq$ $\delta+\sqrt{\delta}$. If $x$ is moreover is positive, then $\operatorname{Re}(\phi(x))+\frac{\delta}{2} \geq 0$.

Proof. The first assertion follows from [24, Lemma 3.1]. The second assertion can be proved in a similar fashion. Indeed, we can assume, without loss of generality, that $Y=\mathbb{C}$, whence $\phi$ is a unital linear functional. Observe that the spectrum $\sigma(x)$ of $x$ is contained in the closed disc of center $\frac{1}{2}$ and radius $\frac{1}{2}$. Therefore $\sigma\left(x-\frac{1}{2}\right)$ is contained in the closed disc of center 0 and radius $\frac{1}{2}$. Hence $\left\|x-\frac{1}{2}\right\| \leq \frac{1}{2}$. It follows that $\left|\phi(x)-\frac{1}{2}\right| \leq \frac{1}{2}(1+\delta)$. 
Lemma 4.2. Suppose that $Y$ is an operator system and $\phi: M_{q} \rightarrow Y$ is a unital linear map such that $\|\phi\|_{q} \leq 1+\delta$. Then there exists a ucp map $\psi: M_{q} \rightarrow Y$ such that $\|\psi-\phi\|_{c b} \leq 3 q^{2} \delta+2 q^{2} \sqrt{\delta}$.

Proof. We can assume, without loss of generality, that $\phi$ is an isometry. Let $b$ be the image under $\phi^{(q)}$ of the matrix $\left[e_{i j}\right] \in M_{q}\left(M_{q}\right)$, where $e_{i j} \in M_{q}$ are the matrix units. Lemma 4.1 shows that there exists a positive element $a \in M_{q}(Y)$ such that $\|b-a\| \leq \frac{3}{2} \delta+\sqrt{\delta}$. If $\psi_{0}: M_{q} \rightarrow A$ is the linear map such that $\psi_{0}\left(e_{i j}\right)=a_{i j}$ then $\psi_{0}$ is completely positive by Choi's theorem [29]. Furthermore by the small perturbation argument, we have $\left\|\psi_{0}-\phi\right\|_{c b} \leq \frac{3}{2} q^{2} \delta+q^{2} \sqrt{\delta}$; see also [13, Proposition 2.40]. Let $\tau$ be the normalized trace on $M_{q}$ and define $\psi: M_{q} \rightarrow Y$ by

$$
\psi(x)=\psi_{0}(x)+\tau(x)\left(\psi_{0}(1)-1\right) .
$$

It follows that $\psi$ is a ucp map such that $\|\psi-\phi\|_{c b} \leq 2\left\|\psi_{0}-\phi\right\|_{c b} \leq 3 q^{2} \delta+2 q^{2} \sqrt{\delta}$.

Observe that, in the previous lemma, if $\left\|\phi^{-1}\right\|_{c b} \leq \frac{1}{1-\delta}$ and $\delta \leq \frac{1}{4}$, then $\|\phi\|_{c b} \leq$ $4 q^{2} \delta+2 q^{2} \sqrt{\delta} \leq 5 q^{2} \sqrt{\delta}$ and $\left\|\psi^{-1}\right\|_{c b} \leq \frac{1}{1-4 q^{2} \delta-2 q^{2} \delta^{2}} \leq \frac{1}{1-5 q^{2} \sqrt{\delta}}$.

Lemma 4.3. Suppose that $Y$ is an operator system and $\phi: M_{q} \rightarrow Y$ is a linear map such that $\|\phi\|_{q} \leq 1+\delta \leq 2$ and $\|\phi(1)-1\| \leq \delta$. Then there exists a ucp map $\psi: M_{q} \rightarrow Y$ such that $\|\psi-\phi\|_{c b} \leq 10 q^{2} \sqrt{\delta}$.

Proof. Let $\phi_{0}: M_{q} \rightarrow Y$ be defined by

$$
x \mapsto \phi(x)+\tau(x)(1-\phi(1)) .
$$

Then $\left\|\phi_{0}-\phi\right\|_{c b} \leq 1+\delta$ and hence $\left\|\phi_{0}\right\|_{c b} \leq\|\phi\|_{c b}+\delta \leq 1+2 \delta \leq 2$. Therefore, by the previous lemma, there exists a ucp map $\psi: M_{q} \rightarrow Y$ such that $\left\|\psi-\phi_{0}\right\|_{c b} \leq 5 q^{2} \sqrt{2 \delta}$ and hence $\|\psi-\phi\|_{c b} \leq 5 q^{2} \sqrt{2 \delta}+\delta \leq 10 q^{2} \sqrt{\delta}$.

Suppose that $X$ is an operator system and $\left(Y_{n}\right)$ is a sequence of operator systems. If $\phi: X \rightarrow \prod_{\mathcal{U}} Y_{n}$ is a ucp map, then a ucp lift for $\phi$ is a sequence $\left(\phi_{n}\right)$ of ucp maps from $X$ to $Y_{n}$ such that, for every $x \in X,\left(\phi_{n}(x)\right)$ is a representative sequence for $\phi(x)$; in formulas $\phi(x)=\left(\phi_{n}(x)\right)^{\bullet}$. Here, and in the rest of this section, all ultrafilters are assumed to be nonprincipal ultrafilters on $\mathbb{N}$.

Corollary 4.4. If $\phi: M_{q} \rightarrow \prod_{\mathcal{U}} Y_{n}$ is a ucp map, then $\phi$ has a ucp lift.

Observe that, by Smith's Lemma [32, Theorem 2.10], if $\phi$ is a complete order embedding and $\left(\phi_{k}\right)$ is a ucp lift for $\phi$, then $\lim _{k \rightarrow \mathcal{U}}\left\|\phi_{k}^{-1}\right\|_{c b}=1$.

Corollary 4.5. If $\phi: \mathbb{G S} \rightarrow \prod_{\mathcal{U}} Y_{n}$ is a ucp map, then $\phi$ has a ucp lift.

Proof. It is observed in [24, $\S 4.3]$ that $\mathbb{G S}$ can be realized as the direct limit of a sequence of full matrix algebras with complete order embeddings as connecting maps. Furthermore, it is a particular case of [25, Theorem 3.3] that, for every $n \in \mathbb{N}$, there exist a complete order embedding $\eta$ of $M_{n}$ into $\mathbb{G S}$ and a ucp projection of $\mathbb{G S}$ onto the range of $\eta$. It follows from these facts and the homogeneity property of $\mathbb{G} \mathbb{S}$ given by [24, Theorem 4.4] that one can find a sequence $\left(X_{k}\right)$ of subsystems of $\mathbb{G S}$ such that, for every $k \in \mathbb{N}$, the following conditions hold:

(1) $\bigcup_{n \geq k} X_{n}$ is dense in $\mathbb{G S}$;

(2) $X_{k}$ is completely order isomorphic to a full matrix algebra;

(3) $X_{k}$ is the range of a ucp projection $P_{k}$ of $\mathbb{G S}$; and

(4) every element of $X_{k}$ is at distance at most $2^{-k}$ from some element of $X_{k+1}$.

For $k \in \mathbb{N}, \phi_{\mid X_{k}}$ is a ucp map and hence, by Corollary 4.4. admits a ucp lift $\left(\phi_{k, n}\right)$. Finally, for $n \in \mathbb{N}$, define $\phi_{n}:=\phi_{n, n} \circ P_{n}$. It remains to observe that $\left(\phi_{n}\right)$ is a ucp lift for $\phi$. 
Corollary 4.6. If $\alpha: \mathbb{G S} \rightarrow \mathbb{G S}^{\mathcal{U}}$ is a complete order embedding, then $\alpha$ has a ucp lift $\left(\alpha_{n}\right)$ made of complete order automorphisms $\alpha_{n}: \mathbb{G S} \rightarrow \mathbb{G S}$. In particular, $\alpha$ is an elementary embedding.

Proof. Let $\left(X_{k}\right)$ be an increasing sequence of subsystems of $\mathbb{G S}$ with dense union such that $X_{k}$ is completely order isomorphic to $M_{n_{k}}$. Let $\left(\phi_{n}\right)$ be a ucp lift of $\alpha$. Then, as mentioned above, for any $k \in \mathbb{N}$, we have $\lim _{n \rightarrow \mathcal{U}}\left\|\left(\phi_{n}\right)_{\mid X_{k}}^{-1}\right\|_{c b}=1$. Therefore, by the homogeneity property of $\mathbb{G S}$, one can find complete order automorphisms $\alpha_{n}: \mathbb{G S} \rightarrow \mathbb{G S}$ such that $\lim _{n \rightarrow \mathcal{U}}\left\|\left(\alpha_{n}-\phi_{n}\right)_{\mid X_{k}}\right\|=0$ for any $k \in \mathbb{N}$. Therefore $\left(\alpha_{n}\right)$ is also ucp lift of $\alpha$.

Remark 4.7. Strongly self-absorbing $\mathrm{C}^{*}$ algebras also enjoy the property that any embedding of them into their ultrapower is elementary. However, they have this property for a completely different reason, namely that any such embedding is unitarily conjugate to the diagonal embedding.

4.2. $\mathbb{G S}$ is the prime model of its theory. The main goal of this subsection is to prove Theorem 4.10, asserting that $\mathbb{G S}$ is the prime model of its theory.

Suppose that $q \leq n$ are positive integers. Let $\vec{a}=\left(a_{0}, \ldots, a_{n^{2}-1}\right)$ be a basis for $M_{n}$ such that $a_{0}=1$ and $\operatorname{span}\left\{a_{0}, \ldots, a_{q^{2}-1}\right\}$ is a subsystem of $M_{n}$ completely order isomorphic to $M_{q}$. Let $\eta_{\vec{a}, q}\left(x_{1}, \ldots, x_{q^{2}-1}\right)$ be the formula

$$
\sup _{\left(\alpha_{0}, \ldots, \alpha_{q^{2}-1}\right) \in X_{\vec{a}, q}} \max \left\{\left\|\sum_{i=0}^{q^{2}-1} \alpha_{i} \otimes x_{i}\right\| \dot{-1,1} \cdot\left\|\sum_{i=0}^{q^{2}-1} \alpha_{i} \otimes x_{i}\right\|\right\}
$$

where $x_{0}=1$ and $X_{\vec{a}, q}$ is the (compact) set of tuples $\alpha_{0}, \ldots, \alpha_{q^{2}-1} \in M_{q}$ such that

$$
\left\|\sum_{i=0}^{q^{2}-1} \alpha_{i} \otimes a_{i}\right\|=1
$$

Let $\theta_{\vec{a}, q}\left(x_{1}, \ldots, x_{q^{2}-1}, y_{1}, \ldots, y_{n^{2}-1}\right)$ be the formula

$$
\max \left\{\eta_{\vec{a}, n}(\vec{y}), \sup _{\left(\alpha_{0}, \ldots, \alpha_{q^{2}-1}\right) \in X_{\vec{a}, q}}\left\|\sum_{i=0}^{q^{2}-1} \alpha_{i} \otimes\left(x_{i}-y_{i}\right)\right\|^{2}-25 q^{4} \eta_{\vec{a}, q}(\vec{x})\right\}
$$

where $y_{0}=1$. Finally let $\sigma_{\vec{a}, q}$ be the sentence

$$
\sup _{\vec{x}} \min \left\{\frac{1}{4}-\eta_{\vec{a}, k}(\vec{x}), \inf _{y_{1}, \ldots, y_{n^{2}}-1} \theta_{\vec{a}, q}(\vec{x}, \vec{y})\right\} .
$$

Lemma 4.8. Suppose that $X$ is an operator system. Then $\sigma_{\vec{a}, q}^{X}=0$ if and only if, for any $\delta \in\left(0, \frac{1}{4}\right]$, any $\varepsilon>0$, and any unital linear $\operatorname{map} \phi: \operatorname{span}\left\{a_{0}, \ldots, a_{q^{2}-1}\right\} \rightarrow X$ such that $\|\phi\|_{c b}<1+\delta$ and $\left\|\phi^{-1}\right\|_{c b}<\frac{1}{1-\delta}$, there exists a ucp map $\psi: M_{n} \rightarrow X$ such that $\left\|\psi^{-1}\right\|_{c b}<1+\varepsilon$ and $\|\psi-\phi\|_{q}<5 q^{2} \sqrt{\delta}$.

Proof. One implication is obvious and the other one follows from Lemma 4.2 .

Remark 4.9. It follows form the homogeneity property of $\mathbb{G S}$ that $\sigma_{\vec{a}, q}^{\mathbb{G S}}=0$.

Theorem 4.10. $\mathbb{G S}$ is the prime model of its theory.

Proof. In light of the fact that by Corollary 4.6 every embedding of $\mathbb{G S}$ into its ultrapower is elementary, it suffices to show that $\mathbb{G S}$ embeds into any model of its theory. Towards this end, fix an operator system $Y$ that is elementarily equivalent to $\mathbb{G S}$. Let $\left(X_{k}\right)$ be a sequence of subsystems of $\mathbb{G S}$ with dense union such that $X_{k}$ is completely 
order isomorphic to $M_{n_{k}}$. Fix also a sequence $\left(\varepsilon_{k}\right)$ of positive real numbers such that $\sum_{k} \varepsilon_{k}<+\infty$. Using the fact that $Y$ is a model of the theory of $\mathbb{G S}$ and the lemma above, one can define by recursion on $k$ ucp maps $\psi_{k}: X_{k} \rightarrow Y$ such that:

(1) $\left\|\psi_{k}^{-1}\right\|_{c b}<1+\varepsilon_{k}$, and

(2) $\left\|\left(\psi_{k+1}\right)_{\mid X_{k}}-\psi_{k}\right\|<\varepsilon_{k}$.

Define $\psi: \mathbb{G S} \rightarrow X$ by setting $\psi(x)=\lim _{k \rightarrow+\infty} \psi_{k}(x)$ for $x \in \bigcup_{k} M_{k}$ and extending by continuity. Observe that $\psi$ is well defined by (2) and it is a complete order embedding by (1).

Remark 4.11. Theorem 4.10 should be compared with [13, Proposition 5.1], asserting that $\mathcal{O}_{2}$ is the prime model of its theory.

4.3. $\mathbb{G S}$ does not have quantifier elimination. In this section, we prove that $\mathbb{G S}$ does not have quantifier elimination. In fact, we offer two (very different) proofs. The first proof relies on the following fact.

Fact 4.12 ([14] $)$. There does not exist a family $\Gamma_{m, n}\left(\vec{x}_{m}\right)$ of definable predicates in the language of operator systems (taking only nonnegative values) for which an operator system $E$ is 1-exact if and only if, for every $m$ and every $\vec{a} \in E^{\vec{x} m}$, we have $\inf _{n} \Gamma_{m, n}^{E}(\vec{a})=0$.

Theorem 4.13. $\mathrm{Th}(\mathbb{G S})$ does not have quantifier-elimination.

Proof. Suppose, towards a contradiction, that $\mathbb{G S}$ has quantifier elimination. For each $m$, let $\left(b_{m, n}\right)_{n}$ denote a countable dense subset of (the unit ball of) $\mathbb{G S}^{m}$. Let $p_{m, n}:=$ $\operatorname{tp}^{\mathbb{G S}}\left(b_{m, n}\right)$. Since $\mathbb{G S}$ is the prime model (whence atomic), each $p_{m, n}$ is isolated, so the predicate $d\left(\cdot, p_{m, n}\right)$ is a definable predicate, meaning that there are formula $\varphi_{m, n}^{k}\left(\vec{x}_{m}\right)$ such that, for all $\vec{a}$ (in a monster model of $\operatorname{Th}(\mathbb{G S})$ ), $d\left(\vec{a}, p_{m, n}\right)=\lim _{k} \varphi_{m, n}^{k}(\vec{a})$ uniformly. Since we are assuming a contrario that $\operatorname{Th}(\mathbb{G S})$ has quantifier elimination, we may further suppose that the formulae $\varphi_{m, n}^{k}$ are quantifier-free. Since any operator system embeds into a model of $\operatorname{Th}(\mathbb{G S})$, we can thus consider the definable (relative to the elementary class of operator systems) predicates $\Gamma_{m, n}\left(\vec{x}_{m}\right):=\lim _{k} \varphi_{m, n}^{k}\left(\vec{x}_{m}\right)$; see [5, Section 3.2].

We obtain the desired contradiction by showing that an operator system $E$ is 1-exact if and only if $\inf _{n} \Gamma_{m, n}^{E}(\vec{a})=0$ for all $m$ and all $\vec{a} \in E^{m}$.

First suppose that $\inf _{n} \Gamma_{m, n}^{E}(\vec{a})=0$ for all $m$ and all $\vec{a} \in E^{m}$. In order to show that $E$ is 1-exact, it suffices to show that all of its finite-dimensional subsystems are 1-exact. Thus, without loss of generality, we may assume that $E$ is the operator system generated by $\vec{a}$ for some linearly independent tuple $\vec{a}$. Fix $M \models \operatorname{Th}(\mathbb{G S})$ containing $E$. Since $\inf _{n} \Gamma_{m, n}^{M}(\vec{a})=\inf _{n} \Gamma_{m, n}^{E}(\vec{a})=0$ (as $\Gamma$ is a quantifier-free definable predicate), we have that $\operatorname{tp}^{M}(\vec{a})$ is in the metric closure of the isolated types, whence is itself isolated. Since isolated types are realized in all models, there is $\vec{b} \in \mathbb{G S}^{m}$ such that $\operatorname{tp}^{M}(\vec{a})=\operatorname{tp}^{\mathbb{G S}}(\vec{b})$. It follows that the map $a_{i} \mapsto b_{i}$ is a complete isometry, whence $E$ is 1-exact.

Conversely, suppose that $E$ is 1-exact. Fix $\vec{a} \in E^{m}$. We must show that $\inf _{n} \Gamma_{m, n}^{E}(\vec{a})=$ 0 . Without loss of generality, we may assume that $E$ is separable, whence we may further assume that $E$ is a subsystem of $\mathbb{G} \mathbb{S}$. Since $\Gamma$ is a quantifier-free definable predicate, we have that $\inf _{n} \Gamma_{m, n}^{E}(\vec{a})=\inf _{n} \Gamma_{m, n}^{\mathbb{G S}}(\vec{a})=0$ as $\left(b_{m, n}\right)$ is dense in $\mathbb{G S}^{m}$.

Corollary 4.14. There is $q \in \mathbb{N}$ such that $\mathbb{G S}$ is not unitally q-isometric to $\mathbb{G}_{q}^{u}$.

Proof. Since any formula in the language of operator systems is a formula in the language of $M_{q}$-systems for some $q$, if $\mathbb{G S}$ were unitally $q$-isometric to $\mathbb{G}_{q}^{u}$ for every $q$, then quantifier-elimination for $\mathbb{G}_{q}^{u}$ would imply quantifier elimination for $\mathbb{G S}$. 
The second proof that $\mathbb{G S}$ does not have quantifier elimination is analogous to the proof given in [11] that shows that $\mathcal{O}_{2}$ does not have quantifier-elimination.

The following lemma can be proved in a manner similar to the proof of [11, Proposition 1.16]; see also [2, Proposition 13.6].

Lemma 4.15. Suppose that $X$ is an operator system. Then the following statements are equivalent:

(1) $X$ has quantifier elimination;

(2) If $Y$ is a separable operator system elementarily equivalent to $X$ and $Y_{0}$ is a subsystem of $Y$, then any complete order embedding of $Y_{0}$ into an ultrapower $X^{\mathcal{U}}$ of $X$ can be extended to a complete order embedding of $Y$ into $X^{\mathcal{U}}$.

A unital $\mathrm{C}^{*}$-algebra $A$ is quasidiagonal if, for every finite subset $F$ of $A$ and every $\varepsilon>0$, there exists $n \in \mathbb{N}$ and a ucp map $\phi: A \rightarrow M_{n}$ such that

$$
\|\phi(a b)-\phi(a) \phi(b)\|<\varepsilon \text { and }\|\phi(a)\|>\|a\|-\varepsilon .
$$

Equivalently $A$ is quasidiagonal if there exits a unital injective ${ }^{*}$-homomorphism $\phi$ : $A \rightarrow M$ that admits a ucp lift, where $M$ is the ultraproduct $\prod_{\mathcal{U}} M_{n}$.

We now can give the second proof that the theory of $\mathbb{G S}$ does not have quantifier elimination. Let $\mathbb{F}_{2}$ be the free group on two generators. By a result of Rosenberg from [17], the reduced $\mathrm{C}^{*}$-algebra $C_{r}^{*}\left(\mathbb{F}_{2}\right)$ is not quasidiagonal. However, by a result of Haagerup and Thorbjorsen from [16], there exists an injective unital *-homomorphism $\phi$ from $C_{r}^{*}\left(\mathbb{F}_{2}\right)$ to the ultraproduct $M:=\prod_{\mathcal{U}} M_{n}$. As mentioned above, it is shown in [25] that, for every $n \in \mathbb{N}$, there exists a complete order embedding $\eta_{n}: M_{n} \rightarrow \mathbb{G S}$ and a ucp projection $P_{n}$ from $\mathbb{G S}$ onto the range of $\eta_{n}$. Let $\eta=\left(\eta_{n}\right)^{\bullet}: M \rightarrow \mathbb{G S}^{\mathcal{U}}$. Then $\eta \circ \phi: C_{r}^{*}\left(\mathbb{F}_{2}\right) \rightarrow \mathbb{G S}$ is a complete order embedding. Since $C_{r}^{*}\left(\mathbb{F}_{2}\right)$ a unital exact $\mathrm{C}^{*}$-algebra, one can regard $C_{r}^{*}\left(\mathbb{F}_{2}\right)$ as a subsystem of $\mathbb{G S}$ by universality. We claim that $\eta \circ \phi$ has no extension to a linear complete isometry $\psi: \mathbb{G S} \rightarrow \mathbb{G S}^{\mathcal{U}}$. Indeed, if $\psi$ is such an extension, then by Corollary 4.5, $\psi$ has a ucp lift $\left(\psi_{n}\right)$, whence the sequence $\left(P_{n} \circ\left(\psi_{n}\right)_{\mid C_{r}^{*}\left(\mathbb{F}_{2}\right)}\right)$ is a ucp lift for $\phi$, contradicting the fact that $C_{r}^{*}\left(\mathbb{F}_{2}\right)$ is not quasidiagonal. One can then conclude that $\mathbb{G S}$ does not have quantifier elimination by applying Lemma 4.15.

Remark 4.16. The first proof that $\mathrm{Th}(\mathbb{G S})$ does not have quantifier elimination relies on the work of Junge and Pisier in 19], which (essentially) shows that the set of $n$ dimensional 1-exact operator systems is not a Polish space in the weak topology. Instead, the second proof uses in an essential way the aforementioned deep result of Haagerup and Thorbjorsen from [16].

4.4. Existentially closed operator systems. Recall that an operator system $X$ is nuclear if there exist nets $\rho_{\alpha}: X \rightarrow M_{n_{\alpha}}$ and $\gamma_{\alpha}: M_{n_{\alpha}} \rightarrow X$ of ucp maps such that $\gamma_{\alpha} \circ \rho_{\alpha}$ converge pointwise to the identity map of $X$; see [18, Theorem 3.1]. We also recall that an operator system $X$ is existentially closed if, whenever $Y$ is an operator system containing $X, \varphi(\vec{x}, y)$ is a quantifier-free formula in the language of operator systems, and $\vec{a}$ is a tuple of elements of $X$, one has that

$$
\inf _{b \in \operatorname{Ball}(Y)} \varphi(\vec{a}, b)=\inf _{b \in \operatorname{Ball}(X)} \varphi(\vec{a}, b) .
$$

The operator system $X$ is positively existentially closed if one merely assumes that Equation (4.1) holds for quantifier-free formulas $\phi$ constructed using only nondecreasing functions as connectives. Similar definitions can be given for operator spaces.

Note that the formulae $\theta_{\vec{a}, q}$ from the proof of Theorem 4.10 are really quantifier-free definable predicates (as the supremum over a compact set is a limit of maxima over finer finite nets). Furthermore the category of operator systems admits approximate pushouts as in [25, Lemma 3.1]. This can be seen as in [25, Lemma 3.1] by replacing 
$M_{q}$ with $B(H)$. It follows that the proof of Theorem 4.10, whith the extra ingredient of approximate pushouts, also shows:

Theorem 4.17. $\mathbb{G S}$ embeds into any existentially closed operator system.

The next two results explore what happens when we combine 1-exactness and existential closedness.

Proposition 4.18. Suppose that $X$ is a 1-exact operator system. Then $X$ is nuclear if and only if it is positively existentially closed.

Proof. Suppose that $X$ is a nuclear operator system. Let $X$ be a subsystem of $Z$. Let $\varphi(\vec{x}, y)$ be a positive quantifier-free formula in the language of operator systems and $\vec{a}$ a tuple in $X$. Suppose that $r>0$ and $b \in Z$ is such that $\varphi(\vec{a}, b)<r$. Fix $\varepsilon>0$ such that $\varphi(\vec{a}, b)+\varepsilon<r$ and $\delta>0$ small enough. Consider the inclusion maps $\phi:\langle\vec{a}\rangle \rightarrow\langle\vec{a}, b\rangle$ and $f:\langle\vec{a}\rangle \rightarrow X$. By the implication $(3) \Rightarrow(1)$ of [25, Lemma 3.3], there exists a ucp map $g:\langle\vec{a}, b\rangle \rightarrow X$ such that $\|g \circ \phi-f\|<\delta$. Thus, for $\delta$ small enough, we have

$$
\varphi(\vec{a}, g(b)) \leq \varphi(g(\phi(\vec{a})), g(b))+\varepsilon=\varphi(\vec{a}, b)+\varepsilon<r .
$$

Suppose now that $X$ is a positively existentially closed operator system. We want to show that $X$ is nuclear. Suppose that $q \in \mathbb{N}, E$ is a subsystem of $M_{q}$, and $f: E \rightarrow X$ is a ucp map. Fix $\varepsilon>0$. We want to show that there exists a ucp map $g: M_{q} \rightarrow X$ such that $\left\|g_{\mid E}-f\right\|<\varepsilon$. This will imply that $X$ is nuclear by the implication $(2) \Rightarrow(3)$ in 25, Lemma 3.3]. Let $\delta>0$ be small enough. Arguing as in the proof of [25, Lemma 3.1] (where one replaces $M_{q}$ with $B(H)$ ) one can show that there exist a ucp map $i: M_{q} \rightarrow$ $B(H)$ and a complete order embedding $j: X \rightarrow B(H)$ such that $\left\|i_{\mid E}-j \circ f\right\|<\delta$. Let $\vec{c}=\left(c_{0}, \ldots, c_{q^{2}-1}\right)$ be a normalized basis of $M_{q}$ such that $\left(c_{0}, \ldots, c_{k-1}\right)$ is a basis of $E$ and let $\vec{a}=\left(f\left(c_{0}\right), \ldots, f\left(c_{k-1}\right)\right)$ and $\vec{b}=\left(g\left(c_{0}\right), \ldots, g\left(c_{q^{2}-1}\right)\right)$. Consider the formula $\eta\left(y_{1}, \ldots, y_{q^{2}-1}\right)$ defined by

$$
\sup _{\left(\alpha_{0}, \ldots, \alpha_{q^{2}-1}\right) \in X_{\vec{a}, q}}\left\|\sum_{i=0}^{q^{2}-1} \alpha_{i} \otimes y_{i}\right\|-1,
$$

where $X_{\vec{a}, q}$ is the (compact) set of tuples $\left(\alpha_{0}, \ldots, \alpha_{q^{2}-1}\right)$ in $M_{q}$ such that

$$
\left\|\sum_{i=0}^{q^{2}-1} \alpha_{i} \otimes a_{i}\right\| \leq 1
$$

Consider then the formula $\theta\left(x_{1}, \ldots, x_{k-1}, y_{1}, \ldots, y_{q^{2}-1}\right)$ defined by

$$
\max \left\{\eta\left(y_{1}, \ldots, y_{q^{2}-1}\right), \sup _{\left(\alpha_{0}, \ldots, \alpha_{k-1}\right) \in X_{\vec{a}, q}}\left\|\sum_{i-0}^{k-1} \alpha_{i} \otimes\left(x_{i}-y_{i}\right)\right\|\right\}
$$

where $x_{0}=y_{0}=1$ and $X_{\vec{a}, q}$ is defined as above. Then $\theta(\vec{a}, \vec{b})=0$; since $X$ is positively existentially closed, there exists a tuple $\vec{d}$ in $X$ such that $\theta(\vec{a}, \vec{d})<\delta$. Let $\psi: M_{q} \rightarrow X$ be the map sending $a_{0}$ to 1 and $a_{i}$ to $d_{i}$ for $i<q^{2}$; then $\psi$ is a unital linear map such that $\|\psi\|_{q}<1+\delta$ and $\left\|\psi_{\mid E}-f\right\|_{q}<\delta$. By Lemma 4.2, one can find a ucp map $g: M_{q} \rightarrow X$ such that $\|g-\psi\| \leq 5 q^{2} \sqrt{\delta}$. This concludes the proof that $X$ is nuclear by [25, Lemma 3.2].

We thus see that being positively existentially closed characterizes nuclear operator systems among the 1-exact operator systems. We now see that being existentially closed characterizes the Gurarij operator system $\mathbb{G S}$ among the separable 1-exact operator systems. 
Theorem 4.19. Suppose that $X$ is a separable 1-exact operator system. Then $X$ is existentially closed if and only if $X$ is completely order isomorphic to $\mathbb{G S}$.

Proof. One can prove that if $X$ is an existentially closed operator system, then $X$ is completely order isomorphic to $\mathbb{G S}$ arguing as in the proof of Proposition 4.18, where one uses [25, Lemma 3.1] and replaces the formula

$$
\sup _{\left(\alpha_{0}, \ldots, \alpha_{q^{2}-1}\right) \in X_{\vec{a}, q}}\left\|\sum_{i=0}^{q^{2}-1} \alpha_{i} \otimes y_{i}\right\| \dot{-1}
$$

with the formula

$$
\sup _{\left(\alpha_{0}, \ldots, \alpha_{q^{2}-1}\right) \in X_{\vec{a}, q}} \max \left\{\left\|\sum_{i=0}^{q^{2}-1} \alpha_{i} \otimes y_{i}\right\| \dot{-1,1}\|\| \sum_{i=0}^{q^{2}-1} \alpha_{i} \otimes y_{i} \|\right\} .
$$

We now show that $\mathbb{G S}$ is existentially closed. Suppose that $X$ is an operator system containing $\mathbb{G S}$. Let $\vec{a}$ be a tuple of elements of $\mathbb{G S}$ and $\varphi(\vec{a}, x)$ be a quantifier-free formula in the language of operator systems. Since $\mathbb{G S}$ is the direct limit of a sequence of full matrix algebras with unital completely isometric connective maps, without loss of generality we can assume that the operator system $\langle\vec{a}\rangle$ generated by the tuple $\vec{a}$ in $\mathbb{G S}$ admits a complete order embedding into $M_{q}$ for some $q \in \mathbb{N}$. We may also assume that $q$ is chosen large enough so that only appear matrix norms up to order $q$ appear in $\varphi$. Suppose that $r \in \mathbb{N}$ and $\varphi(\vec{a}, b)<r$ for some $b \in \operatorname{Ball}(X)$. Consider the complete order embeddings $\langle\vec{a}\rangle \subset \operatorname{MIN}_{q}(\langle\vec{a}, b\rangle)$ (inclusion map) and $f:\langle\vec{a}\rangle \subset \mathbb{G S}$. Fix $\varepsilon>0$ such that $\varphi(\vec{a}, b)+\varepsilon<r$ and fix $\delta>0$ sufficiently small. By the homogeneity property of $\mathbb{G S}$, there exists a complete isometry $g: \operatorname{MIN}_{q}(\langle\vec{a}, b\rangle) \rightarrow \mathbb{G S}$ such that $\left\|g_{\mid\langle\vec{a}\rangle}-f\right\|<\delta$. It is clear that upon choosing $\delta$ small enough one can ensure that

$$
\varphi(\vec{a}, g(b)) \leq \varphi(g(\vec{a}), g(b))+\varepsilon=\varphi(\vec{a}, b)+\varepsilon<r .
$$

This concludes the proof that $\mathbb{G S}$ is existentially closed.

Remark 4.20. Once we have established that $\mathbb{G S}$ is existentially closed, we obtain another proof of the fact that separable nuclear operator systems are positively existentially closed. Indeed, suppose that $X$ is separable and nuclear. By 25, Theorem 3.3] we may assume that $X$ is a subsystem of $\mathbb{G S}$, and there exists a ucp projection $\phi$ of $\mathbb{G S}$ onto $X$. It suffices to show that $X$ is positively existentially closed in $\mathbb{G S}$, that is, whenever $\varphi(\vec{a}, x)$ is a positive quantifier-free formula and $\vec{a}$ is a tuple from $X$, then we have that

$$
\inf _{b \in \operatorname{Ball}(\mathbb{G S})} \varphi(\vec{a}, b)=\inf _{b \in \operatorname{Ball}(X)} \varphi(\vec{a}, b) .
$$

However if $b \in \operatorname{Ball}(\mathbb{G S})$ then $\varphi(\vec{a}, \phi(b)) \leq \varphi(\vec{a}, b)$, whence the desired result follows.

Theorem 3.1 of [11] shows that the theory of $\mathrm{C}^{*}$-algebras does not have a model companion. We now have the same conclusion for the theory of operator systems:

Corollary 4.21. The theory of operator systems does not have a model companion.

Proof. If the theory of operator systems had a model companion, then it would be a model-completion as the class of operator systems satisfies the amalgamation property; see [20, §2] and also the proof of Lemma 3.1 in [25] where one replaces $M_{q}$ with $B(H)$. Since $\mathbb{G S}$ would be a model of the model-completion, we would conclude that $\mathbb{G S}$ has quantifier-elimination, contradicting Theorem 4.13,

Remark 4.22. In a similar manner, one can show that $\mathbb{N} G$ is an existentially closed operator space. However, since we do not know whether or not $\mathbb{N} \mathbb{G}$ has quantifierelimination, it is still open as to whether or not the theory of operator spaces has 
a model companion. Likewise, we do not know if $\mathbb{N G}$ is the only separable 1-exact existentially closed operator space.

Remark 4.23. Theorem 4.19] should be compared with [13, Proposition 2.18] asserting that the Cuntz algebra $\mathcal{O}_{2}$ is the only possible existentially closed exact $\mathrm{C}^{*}$-algebra. By 13, Theorem 3.3], the assertion that $\mathcal{O}_{2}$ is indeed existentially closed is equivalent to a positive solution to the Kirchberg embedding problem [13, §3.1].

4.5. Nuclear models of the theory of $\mathbb{G S}$. It follows from [13, Corollary 2.9] that $\mathcal{O}_{2}$ is the only nuclear model of its theory. A similar assertion holds for the Gurarij operator system $\mathbb{G S}$.

Lemma 4.24. Suppose that $q \in \mathbb{N}, E \subset M_{q}$ is a subsystem of dimension $k$, and $\vec{a}=\left(a_{0}, \ldots, a_{k-1}\right)$ is a normalized basis of $E$ with $a_{0}=1$. Then there exists a sequence of formulas $\theta_{m}\left(x_{1}, \ldots, x_{k-1}\right)$ in the language of operator systems such that the following holds: if $X$ is a nuclear operator system and $\vec{b}=\left(b_{1}, \ldots, b_{k-1}\right)$ is a tuple in the unit ball of $X$, then for every $m \in \mathbb{N}$, if $\theta_{m}^{X}(\vec{b})<\frac{1}{4}$, then the unital linear map $\phi: E \rightarrow X$ such that $\phi\left(a_{i}\right)=b_{i}$ for $1 \leq i \leq k-1$ is invertible and

$$
\max \left\{\|\phi\|_{c b},\left\|\phi^{-1}\right\|_{c b}\right\}-1 \leq \theta_{m}^{X}(\vec{b}) .
$$

Furthermore, if $\phi: E \rightarrow X$ is an invertible unital linear map and $\vec{b}=\phi(\vec{a})$, then

$$
\inf _{m} \theta_{m}^{X}(\vec{b}) \leq \max \left\{\|\phi\|_{c b},\left\|\phi^{-1}\right\|_{c b}\right\}-1 .
$$

Proof. Suppose that $n \geq q$ and $\vec{c}$ is a $k$-tuple in $M_{n}$ with $c_{0}=1$. Denote by $\left(a_{0}^{\prime}, \ldots, a_{k-1}^{\prime}\right)$ the dual basis of $\left(a_{0}, \ldots, a_{k-1}\right)$. Let $L \geq 1$ be such that $\left\|a_{i}^{\prime}\right\| \leq L$ for $i<k$. Denote by $e_{i j}$ for $i, j \leq n$ the canonical matrix units of $M_{n}$, and let $c_{\ell}=\sum_{i j} \lambda_{i j}^{(\ell)} e_{i j} \in M_{n}$ for $0 \leq \ell \leq k-1$. Consider the formula $\mu_{\vec{c}}\left(x_{1}, \ldots, x_{k-1}\right)$ defined by

$$
\inf _{\left[y_{i j}\right] \in C_{n}} \sup _{\left(\alpha_{0}, \ldots, \alpha_{k-1}\right) \in X_{\vec{c}, n}}\left\|\sum_{\ell=0}^{k-1} \alpha_{\ell} \otimes\left(x_{\ell}-\sum_{1 \leq i, j \leq n} \lambda_{i j}^{(\ell)} y_{i j}\right)\right\|
$$

where $x_{0}=1, X_{\vec{c}, n}$ is the (compact) set of $k$-tuples $\left(\alpha_{0}, \ldots, \alpha_{k-1}\right)$ in $M_{n}$ such that

$$
\left\|\sum_{i=0}^{k-1} \alpha_{i} \otimes c_{i}\right\|=1
$$

and $C_{n}$ is the (definable) set of positive elements of norm at most 1 of $M_{n}(X)$. Consider also the formula $\psi_{\vec{c}}\left(x_{1}, \ldots, x_{k-1}\right)$ defined by

$$
\max \left\{\sup _{\left(\alpha_{0}, \ldots, \alpha_{k-1}\right) \in X_{\vec{c}, n}} 1 \cdot\left\|\sum_{i=0}^{k-1} \alpha_{i} \otimes x_{i}\right\|, \mu_{\vec{c}}(\vec{x})\right\}
$$

and the formula $\eta\left(x_{1}, \ldots, x_{k-1}\right)$ defined by

$$
\sup _{\left(\alpha_{0}, \ldots, a_{k-1}\right) \in X_{\vec{a}, n}} \max \left\{\left\|\sum_{i=0}^{k-1} \alpha_{i} \otimes x_{i}\right\| \dot{-1,1}\|\| \sum_{i=0}^{k-1} \alpha_{1} \otimes x_{i} \|\right\}
$$

where $x_{0}=1$ and $X_{\vec{a}, n}$ is the set of tuples $\left(\alpha_{0}, \ldots, \alpha_{k-1}\right)$ in $M_{n}$ such that

$$
\left\|\sum_{i=0}^{k-1} \alpha_{i} \otimes a_{i}\right\|=1
$$

Let $\theta_{\vec{c}}\left(x_{1}, \ldots, x_{k-1}\right)$ be the formula

$$
\eta\left(x_{1}, \ldots, x_{k-1}\right)+100 L k \psi_{\vec{c}}\left(x_{1}, \ldots, x_{k-1}\right) .
$$


We claim that the collection of formulas $\theta_{\vec{c}}$ satisfies the conclusion of the statement. Since the space of formulas with parameters from various $M_{n}$ 's is separable, we can then just replace the formulas $\theta_{\vec{c}}$ with a countable dense set to obtain a sequence of formulas as in the statement.

Suppose thus that $X$ is a nuclear operator system and $\vec{b}$ is a $k$-tuple of elements of $X$ with $b_{0}=0$. Let $\phi: E \rightarrow X$ be the unital linear map such that $\phi\left(a_{i}\right)=b_{i}$ for $i \leq k-1$. Suppose that $n \geq q$ and $\vec{c}$ is a $k$-tuple in $M_{n}$ with $c_{0}=1$ such that $\theta_{\vec{c}}(\vec{b}) \leq \frac{1}{4}$. Fix $\delta \in\left(0, \frac{1}{4}\right)$ and $\varepsilon \in\left(0, \frac{1}{400 k L}\right)$ such that $\eta(\vec{b})<\delta$ and $\psi_{\vec{c}}(\vec{b})<\varepsilon$. It follows from $\eta(\vec{b})<\delta$ that $\|\phi\|_{n}<1+\delta$. Furthermore, since $\delta<\frac{1}{4}$, we have that $\phi$ is invertible and $\left\|\phi^{-1}\right\|_{n}=\left\|\phi^{-1}\right\|_{c b}<1+\delta$. Since $\left\|a_{i}^{\prime}\right\| \leq L$, a straightforward computation shows that $\left\|b_{i}^{\prime}\right\| \leq 2 L$ for every $i \leq k-1$, where $\left(b_{0}^{\prime}, \ldots, b_{k-1}^{\prime}\right)$ is the dual basis of $\vec{b}$. It remains to show that $\|\phi\|_{c b}<1+\delta+100 k L \varepsilon$. Denote by $\gamma: \operatorname{span}(\vec{b}) \rightarrow M_{n}$ the unital linear map such that $\gamma\left(b_{i}\right)=c_{i}$. Observe that, since $\psi_{\vec{c}}(\vec{b})<\varepsilon$, we have that $\|\gamma\|_{c b}<1+\varepsilon$ and there exists a completely positive map $\rho_{0}: M_{n} \rightarrow X$ such that

$$
\left\|\rho_{0} \circ \gamma-\iota\right\|_{n}<\varepsilon
$$

where $\iota$ is the inclusion map of $\operatorname{span}(\vec{b})$ inside $X$. Define $\rho: M_{n} \rightarrow X$ by

$$
\rho_{0}(z)=\rho(z)+\tau(z)(1-\rho(1)),
$$

where $\tau$ is the normalized trace of $M_{n}$, and observe that $\rho$ is a unital completely positive map such that $\left\|\rho-\rho_{0}\right\|_{c b} \leq\|1-\rho(1)\|<\varepsilon$. Observe now that

$$
\|\gamma \circ \phi\|_{c b}=\|\gamma \circ \phi\|_{n} \leq(1+\delta)(1+2 \varepsilon) \leq 1+\delta+4 \varepsilon
$$

and hence $\|\rho \circ \gamma \circ \phi\|_{c b} \leq 1+\delta+4 \varepsilon$. Since $\|\rho \circ \gamma \circ \phi-\phi\|_{n}<\varepsilon$, the small perturbation argument [30, Lemma 2.13.2] shows that $\|\phi\|_{c b}<1+\delta+100 k L \varepsilon$.

Suppose now that $\phi: E \rightarrow X$ is an invertible unital linear map such that $\|\phi\|_{c b}<1+\delta$ and $\left\|\phi^{-1}\right\|_{c b}<1+\delta$. Fix $\delta^{\prime}, \varepsilon>0$ such that $\delta^{\prime}+100 k L \varepsilon<\delta$ and $\max \left\{\|\phi\|_{c b},\left\|\phi^{-1}\right\|_{c b}\right\}<$ $\delta^{\prime}$. Set $\vec{b}=\phi(\vec{a})$. Since $X$ is nuclear, there exist $n \in \mathbb{N}$ and ucp linear maps $\gamma: X \rightarrow M_{n}$ and $\rho: M_{n} \rightarrow X$ such that

$$
\left\|\left(\rho \circ \gamma-i d_{X}\right)_{\mid \phi[E]}\right\|_{n}<\varepsilon .
$$

Therefore $\theta_{\vec{c}}(\vec{b}) \leq \delta^{\prime}+100 k L \varepsilon<\delta$.

Theorem 4.25. Suppose that $X$ is a separable nuclear operator system that is elementarily equivalent to $\mathbb{G S}$. Then $X$ is completely order isomorphic to $\mathbb{G S}$.

Proof. Fix $q \in \mathbb{N}$ and $E \subset M_{q}$ be a subsystem. Suppose that $\vec{a}$ is a normalized basis of $M_{q}$ with $a_{0}=1$ such that $a_{0}, \ldots, a_{k-1}$ is a basis of $E$. Suppose that $\phi: E \rightarrow X$ is a complete order embedding, $\vec{b}=\left(\phi\left(a_{0}\right), \ldots, \phi\left(a_{k-1}\right)\right)$, and $\varepsilon \in\left(0, \frac{1}{4}\right]$. By [24, Proposition 4.2] and Lemma 4.3, in order to prove that $X$ is completely order isomorphic to $\mathbb{G S}$, it is enough to show that there exists a linear map $\psi: M_{q} \rightarrow X$ such that

$$
\max \left\{\left\|\psi_{\mid E}-\phi\right\|,\|\psi\|_{q}-1,\left\|\psi^{-1}\right\|_{q}-1\right\}<10^{3} k \varepsilon^{\frac{1}{2}}
$$

Let $\theta_{m}\left(x_{1}, \ldots, x_{k-1}\right)$ for $m \in \mathbb{N}$ be the formulas obtained from $\left(a_{0}, \ldots, a_{k-1}\right)$ as in Lemma 4.24. Since $X$ is nuclear, there exists $m \in \mathbb{N}$ such that $\theta_{m}^{X}(\vec{b})<\varepsilon$. Set $\theta=\theta_{m}$. Consider the formula $\eta\left(y_{1}, \ldots, y_{q^{2}-1}\right)$ defined by

$$
\sup _{\left(\alpha_{0}, \ldots, a_{q^{2}-1}\right) \in X_{\vec{a}, q}} \max \left\{\left\|\sum_{i=0}^{q^{2}-1} \alpha_{i} \otimes y_{i}\right\| \dot{-1,1}\|\| \sum_{i=0}^{q^{2}-1} \alpha_{1} \otimes y_{i} \|\right\}
$$


where $y_{0}=1$ and $X_{\vec{a}, q}$ is the set of $q^{2}$-tuples $\left(\alpha_{0}, \ldots, \alpha_{q^{2}-1}\right)$ in $M_{q}$ such that

$$
\left\|\sum_{i=0}^{q^{2}-1} \alpha_{i} \otimes a_{i}\right\|=1
$$

Consider also the formula $\tau\left(x_{1}, \ldots, x_{k-1}, y_{1}, \ldots, y_{q^{2}-1}\right)$ defined by

$$
\inf _{y_{1}, \ldots, y_{q^{2}-1}} \max \left\{\sup _{\left(\alpha_{0}, \ldots, \alpha_{k-1}\right) \in X_{\vec{a}, n}}\left\|\sum_{i=0}^{k-1} \alpha_{i} \otimes\left(x_{i}-y_{i}\right)\right\|^{2}-10^{4} k^{2} \theta(\vec{x}), \eta(\vec{y})\right\}
$$

where $x_{0}=y_{0}=1$ and $X_{\vec{a}, n}$ is the set of $k$-tuples $\left(\alpha_{0}, \ldots, \alpha_{k-1}\right)$ in $M_{n}$ such that

$$
\left\|\sum_{i=0}^{k-1} \alpha_{i} \otimes a_{i}\right\|=1
$$

Let now $\sigma$ be the sentence

$$
\sup _{x_{1}, \ldots, x_{k-1}} \min \left\{\theta(\vec{x})-\frac{1}{4}, \inf _{y_{1}, \ldots, y_{q^{2}}-1} \tau(\vec{x}, \vec{y})\right\} .
$$

Observe that $\sigma^{\mathbb{G S}}=0$ by Lemma 4.24 and the homogeneity property of $\mathbb{G S}$ given by [24, Theorem 4.4]. Since $X$ is elementarily equivalent to $\mathbb{G S}$, we have that $\sigma^{X}=0$. Therefore, there exists a tuple $\vec{c}$ in $X$ such that $\tau(\vec{b}, \vec{c})<\varepsilon$. Let now $\psi: M_{q} \rightarrow X$ be the unital linear map such that $\psi\left(a_{i}\right)=c_{i}$ for $i<q^{2}$. It follows from the fact that $\tau(\vec{b}, \vec{c})<\varepsilon$ that $\psi$ satisfies Equation (4.2).

In [13], it is asked whether $\mathcal{O}_{2}$ is the only exact model of its theory; we also do not know if $\mathbb{G S}$ is the only 1 -exact model of its theory.

\section{Existentially Closed $\mathrm{C}^{*}$-Algebras}

As mentioned earlier, it was proven by the second-named author in [24, §4.6] that $\mathbb{G S}$ is not completely order isomorphic to a $\mathrm{C}^{*}$-algebra. Here we generalize this result by showing that no unital $\mathrm{C}^{*}$-algebra is existentially closed as an operator system.

Lemma 5.1. Suppose that $\phi: X \rightarrow Y$ is a complete order embedding between operator systems. Further suppose that $X$ is existentially closed and $u \in X$ is a unitary. Then $\phi(x)$ is a unitary.

Proof. Suppose that $n \in \mathbb{N}$ and consider the formula $\varphi(u, x)$ defined by

$$
\min \left\{\left\|\left[u \otimes I_{n} \quad x\right]\right\|^{2},\left\|\left[\begin{array}{c}
u \otimes I_{n} \\
x
\end{array}\right]\right\|^{2}\right\}-\|x\|^{2} .
$$

Observe that

$$
\left(\inf _{\|x\| \leq 1} \varphi(u, x)\right)^{X}=2
$$

by [8, Theorem 2.4]. Therefore

$$
\left(\inf _{\|x\|=1} \varphi(\phi(u), x)\right)^{Y}=2,
$$

whence $\phi(u)$ is a unitary of $Y$.

A first draft of this paper contained a proof of the next lemma. We thank Thomas Sinclair for pointing out to us that this lemma follows immediately from Pisier's Linearization Trick (see, for example, [28, Theorem 19]). 
Lemma 5.2. Suppose that $\phi: A \rightarrow B$ is a ucp map between unital $C^{*}$-algebras that maps unitaries to unitaries. Then $\phi$ is a $*$-homomorphism.

We thank Thomas Sinclair for providing a proof for the following lemma.

Lemma 5.3. Suppose that $A$ is a unital $C^{*}$-algebra and $\operatorname{dim}(A)>1$. Then there is a unital $C^{*}$-algebra $B$ and a complete order embedding $\phi: A \rightarrow B$ that is not a *-homomorphism.

Proof. We first remark that $A$ has a nonpure state. Indeed, since the states separate points and every state is a linear combination of pure states, we have that the pure states separate points. Since $\operatorname{dim}(A)>1$, this implies that there are at least two pure states, whence any proper convex combination of these two pure states is nonpure.

Secondly, we remark that a nonpure state on $A$ is not multiplicative. Indeed, if $\phi$ is a proper convex combination of the distinct pure states $\phi_{1}$ and $\phi_{2}$, then taking a unitary $u$ on which $\phi_{1}$ and $\phi_{2}$ differ, we have that $\phi(u)$ has modulus strictly smaller than 1 .

We are now ready to prove the lemma. Suppose that $A$ is concretely represented as a subalgebra of $B(H)$. Let $\phi$ be a non-pure state. Then the map

$$
x \mapsto(\phi(x) \cdot 1) \oplus x: A \rightarrow B(H \oplus H)
$$

is a complete order embedding that is not a $*$-homomorphism.

Corollary 5.4. No unital $C^{*}$-algebra is existentially closed as an operator system.

Proof. This follows immediately from Lemmas 5.1, 5.2, and 5.3 (noting that existentially closed operator systems are infinite-dimensional).

Remark. Lemma 5.1 remains valid in the operator space category as well (with an identical proof). As a consequence, we see that if $Z$ is an existentially closed operator space, then $Z$ has no unitaries. Indeed, if $Z$ is concretely represented as a subspace of $\mathcal{B}(H)$, then the map

$$
x \mapsto x \oplus 0: Z \rightarrow \mathcal{B}(H \oplus H)
$$

is a complete isometric embedding into a $\mathrm{C}^{*}$-algebra whose image contains no unitaries, whence, by Lemma $1, Z$ cannot contain any unitaries. In particular, we see that $\mathbb{N G}$ contains no unitaries, a fact already observed (implicitly) in [27, Proposition 3.2].

Remark. Corollary 5.4 in particular shows that no unital exact $\mathrm{C}^{*}$-algebra $A$ is existentially closed as an operator system. We can be a bit more precise about how $A$ fails to be existentially closed as an operator system. Indeed, since $A$ is exact, by universality, there is a complete order embedding $A \hookrightarrow \mathbb{G S}$. We claim that this embedding is not existential. Indeed, since $\mathbb{G S}$ is existentially closed, if the above embedding were existential, then $A$ would be existentially closed as an operator system, contradicting Corollary 5.4.

Given the above discussion, the following question seems natural:

Question 5.5. Is the class of operator systems unitally completely order isomorphic to a $C^{*}$-algebra an elementary class?

We now give a condition that would ensure a positive answer to Question 5.5. Suppose that $\left(X_{i}: i \in I\right)$ is a family of operator systems and $\mathcal{U}$ is an ultrafilter on $I$. If $u_{i} \in X_{i}$ is a unitary for each $i$, then it is clear that $\left(u_{i}\right)^{\bullet} \in \prod_{\mathcal{U}} X_{i}$ is a unitary of $\prod_{\mathcal{U}} X_{i}$.

Question 5.6. With the preceding notation, if $u$ is a unitary in $\prod_{\mathcal{U}} X_{i}$, are there unitaries $u_{i} \in X_{i}$ for which $u=\left(u_{i}\right)^{\bullet}$ ?

We should note that the analog of Question 5.6 for $\mathrm{C}^{*}$-algebras has a positive answer (see [12]). 
Proposition 5.7. If Question 5.6 has a positive answer, then Question 5.5 has a positive answer.

Proof. Clearly the class of operator systems completely order isomorphic to a $\mathrm{C}^{*}$-algebra is closed under isomorphisms and ultraproducts. It suffices to check that it is closed under ultraroots. Towards this end, suppose that $X$ is an operator system for which $X^{\mathcal{U}}$ is a $\mathrm{C}^{*}$-algebra; we need to show that $X$ is a $\mathrm{C}^{*}$-algebra. It suffices to show that $X$ is closed under multiplication. We first show that the product of any two unitaries in $X$ remains in $X$. Suppose that $u, v \in X$ are unitaries. By [8], $u v \in X$ if and only if the matrix $\left[\begin{array}{ll}1 & u \\ v & x\end{array}\right]$ is $\sqrt{2}$ times a unitary of $M_{2}(X)$. However, the aforementioned matrix is $\sqrt{2}$ times a unitary $A$ of $M_{2}\left(X^{\mathcal{U}}\right)$; by assumption, $A=\left(A_{n}\right)^{\bullet}$, where each $A_{n}$ is a unitary in $M_{2}(X)$. Since unitaries in an operator space form a closed set, we have the desired conclusion.

In order to finish the proof, it suffices to prove that the linear span of the unitaries in $X$ are dense in $X$. Towards this end, fix $x \in X$ with $\|x\| \leq \frac{1}{2}$. By [6, §II.3.2.16], there are unitaries $u_{1}, \ldots, u_{5} \in X^{\mathcal{U}}$ for which $x=\frac{1}{5}\left(u_{1}+\cdots+u_{5}\right)$. By assumption, we may write each $u_{i}=\left(u_{i, n}\right)^{\bullet}$, where each $u_{i, n}$ is a unitary of $X$. It follows that some subsequence of $\left(\frac{1}{5}\left(u_{1, n} \cdots+u_{5, n}\right)\right)$ converges to $x$.

\section{REFERENCES}

1. Itaï Ben Yaacov, Fraïssé limits of metric structures, Journal of Symbolic Logic, to appear.

2. Itaï Ben Yaacov, Alexander Berenstein, C. Ward Henson, and Alexander Usvyatsov, Model theory for metric structures, Model theory with applications to algebra and analysis. Vol. 2, London Mathematical Society Lecture Note Series, vol. 350, Cambridge University Press, 2008, p. 315-427.

3. Itaï Ben Yaacov and C. Ward Henson, Generic orbits and type isolation in the Gurarij space, arXiv:1211.4814 (2012), arXiv: 1211.4814.

4. Itaï Ben Yaacov and Todor Tsankov, Weakly almost periodic functions, modeltheoretic stability, and minimality of topological groups, arXiv:1312.7757 (2013).

5. Itaï Ben Yaacov and Alexander Usvyatsov, Continuous first order logic and local stability, Transactions of the American Mathematical Society 362 (2010), no. 10, 5213-5259.

6. Bruce Blackadar, Operator algebras, Encyclopaedia of Mathematical Sciences, vol. 122, Springer-Verlag, Berlin, 2006.

7. David P. Blecher and Christian Le Merdy, Operator algebras and their modules - an operator space approach, London Mathematical Society Monographs. New Series, vol. 30, Oxford University Press, Oxford, 2004.

8. David P. Blecher and Matthew Neal, Metric characterizations of isometries and of unital operator spaces and systems, Proceedings of the American Mathematical Society 139 (2011), no. 3, 985-998.

9. Nathanial P. Brown and Narutaka Ozawa, $C^{*}$-algebras and finite-dimensional approximations, Graduate Studies in Mathematics, vol. 88, American Mathematical Society, 2008.

10. Man Duen Choi, Completely positive linear maps on complex matrices, Linear Algebra and its Applications 10 (1975), 285-290.

11. Christopher J. Eagle, Ilijas Farah, Eberhard Kirchberg, and Alessandro Vignati, Quantifier elimination in $C^{*}$-algebras, arXiv:1502.00573 (2015).

12. Ilijas Farah, Bradd Hart, Martino Lupini, Leonel Robert, Aaron P. Tikuisis, Alessandro Vignati, and Wilhelm Winter, Model theory of nuclear $C^{*}$-algebras, In preparation. 
13. Isaac Goldbring and Thomas Sinclair, On Kirchberg's embedding problem, Journal of Functional Analysis, to appear.

14. _ Omitting types in operator systems, arXiv:1501.06395 (2015).

15. Vladimir I. Gurariǔ, Spaces of universal placement, isotropic spaces and a problem of Mazur on rotations of Banach spaces, Siberian Mathematical Journal 7 (1966), $1002-1013$.

16. Uffe Haagerup and Steen Thorbjørnsen, A new application of random matrices: $\operatorname{Ext}\left(C_{\text {red }}^{*}\left(F_{2}\right)\right)$ is not a group, Annals of Mathematics. Second Series 162 (2005), no. $2,711-775$.

17. Don Hadwin, Strongly quasidiagonal $C^{*}$-algebras, Journal of Operator Theory 18 (1987), no. 1, 3-18.

18. Kyung Hoon Han and Vern I. Paulsen, An approximation theorem for nuclear operator systems, Journal of Functional Analysis 261 (2011), no. 4, 999-1009.

19. Marius Junge and Gilles Pisier, Bilinear forms on exact operator spaces and $B(H) \otimes$ $B(H)$, Geometric and Functional Analysis 5 (1995), no. 2, 329-363.

20. David Kerr and Hanfeng Li, On Gromov-Hausdorff convergence for operator metric spaces, Journal of Operator Theory 62 (2009), no. 1, 83-109.

21. Wiesław Kubiś and Sławomir Solecki, A proof of uniqueness of the Gurariu space, Israel Journal of Mathematics 195 (2013), no. 1, 449-456.

22. Franz Lehner, $M_{n}$-espaces, sommes d'unitaires et analyse harmonique sur le groupe libre, Ph.D. thesis, Université de Paris 6, 1997.

23. Martino Lupini, Uniqueness, universality, and homogeneity of the noncommutative Gurarij space, arXiv:1410.3345 (2014).

24. __ A universal nuclear operator system, arXiv:1412.0281 (2014).

25. ding theorem, arXiv:1502.05966 (2015).

26. Wolfgang Lusky, The Gurarij spaces are unique, Archiv der Mathematik 27 (1976), no. $6,627-635$.

27. Timur Oikhberg, The non-commutative Gurarii space, Archiv der Mathematik 86 (2006), no. 4, 356-364.

28. Narutaka Ozawa, About the Connes embedding conjecture: algebraic approaches, Japanese Journal of Mathematics 8 (2013), no. 1, 147-183.

29. Vern Paulsen, Completely bounded maps and operator algebras, Cambridge Studies in Advanced Mathematics, vol. 78, Cambridge University Press, Cambridge, 2002.

30. Gilles Pisier, Introduction to operator space theory, London Mathematical Society Lecture Note Series, vol. 294, Cambridge University Press, Cambridge, 2003.

31. Zhong-Jin Ruan, Subspaces of $C^{*}$-algebras, Journal of Functional Analysis $\mathbf{7 6}$ (1988), no. 1, 217-230.

32. Roger R. Smith, Completely bounded maps between $C^{*}$-Algebras, Journal of the London Mathematical Society s2-27 (1983), no. 1, 157-166.

33. Blerina Xhabli, The super operator system structures and their applications in quantum entanglement theory, Journal of Functional Analysis 262 (2012), no. 4, 14661497.

Isaac Goldbring, Department of Mathematics, Statistics, and Computer Science, University of Illinois at Chicago, Science and Engineering Offices M/C 249, 851 S. Morgan St., Chichgo, IL, 60607-7045

E-mail address: isaac@math.uic.edu

$U R L:$ http://www.math.uic. edu/ ${ }^{\sim}$ isaac

Fakultät für Mathematik, Universität Wien, Oskar-Morgenstern-Platz 1, Room 02.126, 1090 Wien, Austria.

E-mail address: martino.lupini@univie.ac.at

$U R L$ : http://www.lupini.org/ 